PAPER

\section{On the anodic aluminium oxide refractive index of nanoporous templates}

To cite this article: A Hierro-Rodriguez et al 2015 J. Phys. D: Appl. Phys. 48455105

View the article online for updates and enhancements.
Related content

- Hydrogen sensing via anomalous optical absorption of Pd-based metamaterials Rodrigues et al.

Anodic aluminum oxide with fine pore size control for selective and effective

particulate matter filtering

Su Zhang, Yang Wang, Yingling Tan et al.

Cyclic voltammetry as a tool to estimate the effective pore density of an anodic aluminiumoxide template

Xiaoli Cui, Qiang Zhao, Zhizhou Li et al.

\section{Recent citations}

Current Trends in Nanoporous Anodized Alumina Platforms for Biosensing Applications

Ganesan Sriram et al 


\title{
On the anodic aluminium oxide refractive index of nanoporous templates
}

\author{
A Hierro-Rodriguez ${ }^{1,2}$, P Rocha-Rodrigues ${ }^{1}$, F Valdés-Bango ${ }^{3,4}$, \\ J M Alameda ${ }^{3,4}$, P A S Jorge ${ }^{2}$, J L Santos ${ }^{2,5}$, J P Araujo ${ }^{1,5}$, J M Teixeira ${ }^{3,6}$ \\ and A Guerreiro ${ }^{2,5}$ \\ ${ }^{1}$ IFIMUP and IN, Institute of Nanoscience and Nanotechnology, Rua do Campo Alegre, 4169-007 Porto, \\ Portugal \\ 2 INESC-TEC (Coordinated by INESC-Porto), Rua do Campo Alegre, 4169-007 Porto, Portugal \\ 3 Depto. Física, Fac. Ciencias, Universidad de Oviedo, Avda. Calvo Sotelo s/n, 33007 Oviedo, Spain \\ ${ }^{4}$ Centro de Investigación en Nanomateriales y Nanotecnología-CINN (CSIC-Univ. de Oviedo- \\ Principado de Asturias), 33940 El Entrego, Spain \\ 5 Departamento de Física e Astronomia, Faculdade de Ciências, Universidade do Porto, Rua Campo \\ Alegre, 4169-007 Porto, Portugal \\ ${ }^{6}$ Past address: IFIMUP and IN, Institute of Nanoscience and Nanotechnology, Rua do Campo Alegre, \\ 4169-007 Porto, Portugal \\ E-mail: ahierro@fc.up.pt
}

Received 15 June 2015, revised 7 September 2015

Accepted for publication 14 September 2015

Published 13 October 2015

\begin{abstract}
In the present study, we have determined the intrinsic refractive index of anodic aluminium oxide, which is originated by the formation of nanoporous alumina templates. Different templates have been fabricated by the conventional two-step anodization procedure in oxalic acid. Their porosities were modified by chemical wet etching allowing the tuning of their effective refractive indexes (air-filled nanopores + anodic aluminium oxide). By standard spectroscopic light transmission measurements, the effective refractive index for each different template was extracted in the VIS-NIR region. The determination of the intrinsic anodic aluminium oxide refractive index was performed by using the Maxwell-Garnett homogenization theory. The results are coincident for all the fabricated samples. The obtained refractive index $(\sim 1.55)$ is quite lower $(\sim 22 \%)$ than the commonly used $\mathrm{Al}_{2} \mathrm{O}_{3}$ handbook value $(\sim 1.75)$, showing that the amorphous nature of the anodic oxide structure strongly conditions its optical properties. This difference is critical for the correct design and modeling of optical plasmonic metamaterials based on anodic aluminium oxide nanoporous templates.
\end{abstract}

Keywords: nanoporous templates, anodic aluminium oxide, refractive index

(Some figures may appear in colour only in the online journal)

\section{Introduction}

Optical metamaterials are attracting increasing attention from different research areas such as physics, life sciences, engineering, and material sciences $[1,2]$. This growing interest is due to their great potential in the development of novel materials with exotic optical properties, which can be useful in a very broad interdisciplinary spectrum. Like in other kinds of nanostructured systems, the metamaterials' optical behaviour arises from the combination of both the shapes and order of nanostructures, and the intrinsic constituent materials' properties (usually combining dielectric and metallic materials). Hence, their responses can be completely tuned by properly selecting and carefully designing the materials and structures, respectively [3]. They exhibit bizarre phenomena like negative refraction [4], hyperbolic dispersion [5], and superlensing [6] to name a few, and their possible applications range from nondiffraction-limited imaging [7] and enhanced plasmonic-based sensing $[8,9]$ up to the development of optical cloaking devices $[10,11]$. The future impact of optical metamaterials in society 
will not be only conditioned by their potential applications, but also by their implementation costs. Therefore, moving to bottom-up nanofabrication approaches seems to be a better choice than conventional top-down ones for the successful industrial-scale production of optical metamaterials due to their inherent low costs and large area fabrication capabilities. In this framework, nanoporous anodic aluminium oxide (AAO) membranes are self-assembled systems which can be used as templates for fabricating a wide variety of nanostructured materials with different purposes. By combining the membranes with different fabrication methods, such as physical vapour deposition, etching, and electrodeposition to name a few, a huge variety of nanostructured 2D and 3D systems can be prepared [12-14]. Their applications range from magnetic [15-18] to biomedical ones $[19,20]$, including optical metamaterials $[9,21,22]$. The latter consist generally of metallic nanowires embedded inside the AAO template. Thus, to properly design and simulate the resulting metamaterials' behaviour, the intrinsic optical properties of the AAO must be well established.

This work aims to extract the intrinsic AAO refractive index at the end of visible and NIR spectral regions by performing spectroscopic light transmission measurements in three different AAO templates. The samples have been fabricated following the same process but changing their natural porosities at the end. This allows the tuning of the effective refractive index of the AAO template due to the increase in the empty nanopores' volume. The calculation of the intrinsic AAO refractive index is performed in two steps. First, the effective refractive index of the templates is calculated by directly fitting the Fabry-Pérot interference pattern of each sample, and second by using the Maxwell-Garnett homogenization theory in combination with an accurate scanning electron microscopy (SEM) micrograph analysis of each template. The obtained results for all the samples are coincident leading to a value of $n_{\mathrm{AAO}} \approx 1.55$, which is quite lower $(\sim 22 \%)$ than the commonly used $\mathrm{Al}_{2} \mathrm{O}_{3}$ handbook refractive index of 1.75 [23] and also lower than the $\mathrm{Al}_{2} \mathrm{O}_{3}$ value of $\sim 1.63$ in sputterdeposited thin films [24]. These differences are critical and must be taken into account when designing AAO templatebased optical metamaterials, especially when dealing with plasmonic effects due to the strong dependence of the plasmonic modes with the refractive index [9].

\section{Experimental details}

\section{1. $A A O$ template fabrication}

Three different pairs of AAO templates have been fabricated following the well-known two-step anodization protocol [12, $13,25]$ in the same electrochemical cells and at the same time. The process begins with the anodization of high purity (99.997\%) aluminium foil in a $0.3 \mathrm{M}$ oxalic acid solution under a constant potential of $40 \mathrm{~V}$ and constant temperature $\left(\sim 4{ }^{\circ} \mathrm{C}\right)$. This first anodization takes place for $24 \mathrm{~h}$, and the resulting AAO templates are chemically removed to perform a second $8 \mathrm{~h}$ anodization process under the same conditions. The latter greatly improves the nanopores' order and determines the thickness of the final AAO template. Consequently, a pore
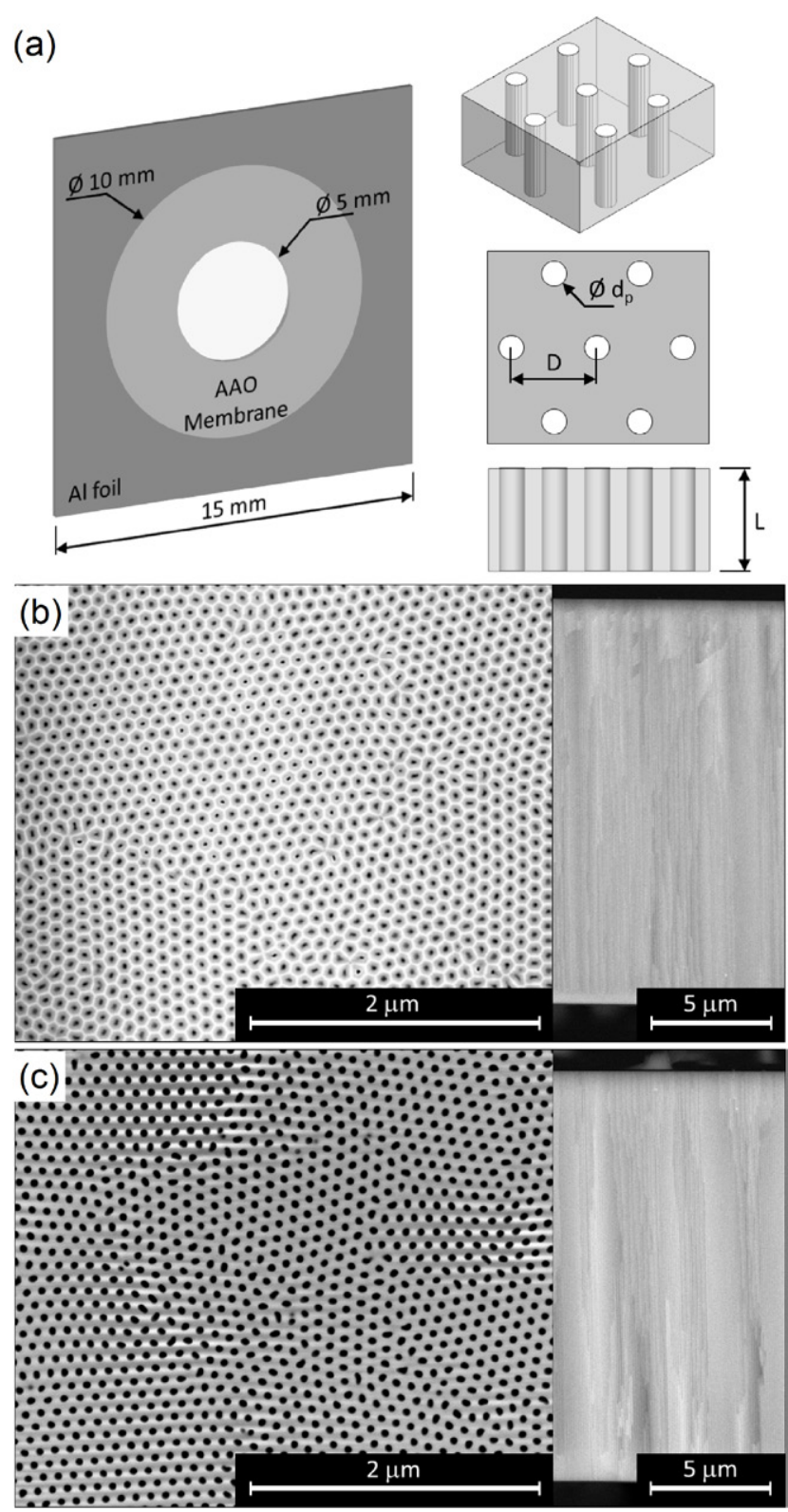

Figure 1. (a) Final sample scheme showing the macroscopic shape and main microscopic structural parameters: inter-pore distance $(D)$, pore diameter $\left(d_{\mathrm{p}}\right)$ and AAO template thickness $(L)$. Top and cross-section views of the SEM micrographs for the AAO_01 (b) and AAO_03 (c) samples.

widening process was performed in two of the three pairs of samples to change their natural porosities by increasing the volume of the nanopores. This process consists of exposing the AAO templates to a $0.5 \mathrm{M}$ phosphoric acid solution at $30{ }^{\circ} \mathrm{C}$. Wider nanopores were obtained by increasing the etching time. The fabricated template pairs are designated as AAO_01, AAO_02, and AAO_03, where the first one was kept with its natural porosity, and the second and third ones were etched for $4 \mathrm{~min}$ and $12 \mathrm{~min}$, respectively.

Following the pore widening, the samples were prepared for light transmission measurements by selectively wet etching a hole in the Al foil substrate and dry etching the AAO barrier layer present at the bottom of the nanopores $\left(\mathrm{Ar}^{+}\right.$ion milling in a Kenosistec RIE/RIBE KP 600 PBS). Thus, at 


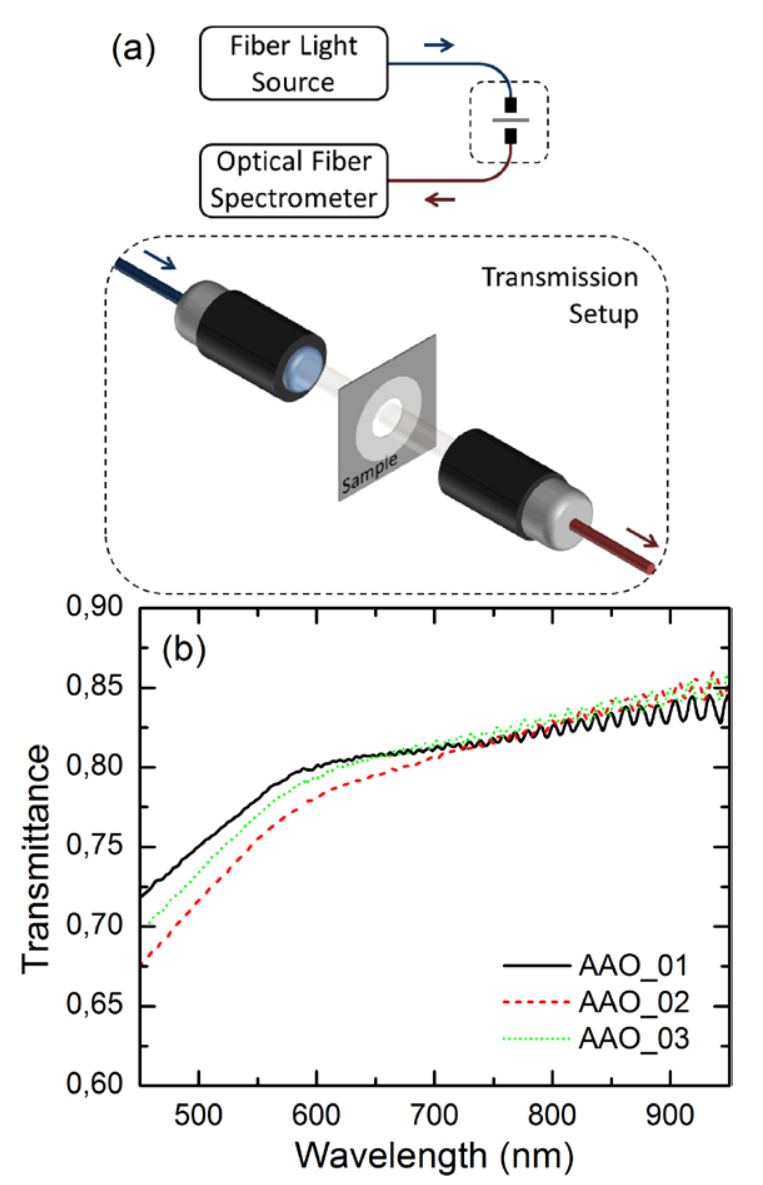

Figure 2. (a) Experimental setup scheme for the spectroscopic light transmission measurements at normal incidence. (b) Transmittance spectra for the three fabricated AAO templates, AAO_01 (black line), AAO_02 (red line) and AAO_03 (green line).

the end, three pairs of AAO templates with different porosities were fabricated from oxalic acid $\mathrm{Al}$ foil anodization with almost the same thickness and with open pores at the top and bottom sides. One sample of each pair was destructively analysed by SEM to obtain both top and cross-section views of the templates, allowing the characterization of the main structural parameters, namely the interpore distance $(D)$, the nanopores' diameters $\left(d_{\mathrm{p}}\right)$, and the template thicknesses $(L)$ (figure 1). Figures 1(b) and (c) present the top and cross-sections views of the AAO_01 and AAO_03 samples, respectively, showing the effect of the pore widening process $\left(\left\langle d_{\mathrm{p}}\right\rangle \approx 27 \mathrm{~nm}\right.$ and $50 \mathrm{~nm}$, respectively) and almost the same template thicknesses ( $L \approx 17.5 \mu \mathrm{m}$ ). The local contrast changes (dark and bright lines) close to the nanopores in the SEM image of the AAO_03 sample are due to charge effects during the scanning, and they are only related to the relative orientation between the nanopores' lattice and the fast scanning direction of the microscope.

\subsection{Optical characterization}

The spectroscopic light transmission measurements were performed by using a homemade set up consisting of an fibre optic white light source (Ocean Optics HL-2000), two fibre optic collimators (Ocean Optics 74-VIS), and a VIS-NIR fibre optic spectrometer (Ocean Optics USB-4000) (figure 2(a)).
The light is guided by a fibre from the lamp to the injection collimator (blue fibre). After this, it is collected by the second collimator and injected again into a fibre (red fibre), which is connected to the spectrometer at the end. It is important to note here that the diameter of the hole $(5 \mathrm{~mm})$ performed in the $\mathrm{Al}$ foil is larger than the diameter of the collimated light beam $(\sim 3 \mathrm{~mm})$, and also that all the spectra were taken at normal incidence. The transmittance spectra of the samples were obtained by direct comparison between the reference lamp spectrum (measured without the sample) and the templates' spectra. The spectrometer acquisition conditions for both the reference and sample measurements were the same.

The measured transmittance spectra are presented in figure 2(b) for the three fabricated AAO templates in the VISNIR region. All of them show almost the same behaviour: a transmittance level around $80 \%$ with two different regions. For wavelengths below $575 \mathrm{~nm}$, a faster decay in the transmittance is observed, and above this a Fabry-Pérot interference appears mounted over the recorded signal. Both the relatively low transmittance level compared with an $\mathrm{Al}_{2} \mathrm{O}_{3}$ thin film (estimated around 94\%) and the rough behaviour change for smaller wavelengths can be understood in terms of losses due to the light scattering induced by the nanopores and by the different grains, where the nanopores' lattice presents the same arrangement. In fact, this scattering strongly depends on the wavelength and it has been observed that its effects are enhanced for smaller wavelengths [26]. On the other hand, the Fabry-Pérot interference is generated by internal reflections of light passing through the sample and it depends on the effective refractive index of the AAO template (air filled nanopores $+\mathrm{AAO})$ and on the sample's thickness.

\section{Results' analysis and discussion}

The measured AAO refractive index is for $0.3 \mathrm{M}$ oxalic acid $\mathrm{Al}$ foil anodization at $40 \mathrm{~V}$ and $4{ }^{\circ} \mathrm{C}$. Changing the electrolyte and/or the anodizing conditions [27] may change the intrinsic refractive index of the resulting AAO. At this point it is important to emphasize the difference between the effective refractive index $\left(n_{\text {eff }}\right)$ of the templates and the intrinsic refractive index of the AAO. The first one results from the mixture of the air-filled nanopores and the AAO which forms the template. It is dependent on the porosity of the sample and diminishes when the volume of the nanopores increases. The intrinsic AAO refractive index corresponds only to the aluminium oxide regions of the sample and it can be extracted from the $n_{\text {eff }}$ value by using the Maxwell-Garnett homogenization theory in combination with a careful image analysis of the SEM micrographs.

\subsection{Templates' effective refractive index}

The calculation of the effective refractive index of each AAO template was performed by directly fitting the Fabry-Pérot signal of each transmittance spectrum. As indicated before, the resulting signal depends mainly on the template thickness and on the effective refractive index. A dispersive Sellmeier model [28] for the refractive index was constructed within the 
Fabry-Pérot equation to perform the fitting by standard nonlinear least squares using MATLAB.

$$
\begin{aligned}
& M(\lambda)=f(\lambda) \\
& \quad \cdot \sin \left(\frac{2 \pi}{\lambda} \cdot 2 L \sqrt{1+\frac{B_{1} \lambda^{2}}{\lambda^{2}-C_{1}{ }^{2}}+\frac{B_{2} \lambda^{2}}{\lambda^{2}-C_{2}{ }^{2}}+\frac{B_{3} \lambda^{2}}{\lambda^{2}-C_{3}{ }^{2}}}+\delta\right) .
\end{aligned}
$$

Equation (1) shows the constructed fitting model. The function $f(\lambda)$ takes into account the amplitude changes of the oscillating signal with wavelength. This is a second-order polynomial function without an independent term, which was previously optimized and which was fixed during the final fitting. The $B_{i}$ and $C_{i}$ parameters correspond to the amplitudes and the absorption resonance wavelengths of each Sellmeier term, respectively, $L$ represents the thickness of the template, and $\delta$ is an arbitrary initial phase. The initial values for the three different Sellmeier's absorption resonances were established following the method described in [29], and the measured template's thickness was kept fixed during the fit.

The different fitted functions are shown in figure 3(a) for the three fabricated templates. The fitting range was adapted between 700 and $950 \mathrm{~nm}$, where the Fabry-Pérot signal is good enough to perform the calculation. All the fits present a $\chi^{2}$ superior to 0.9 , matching fairly well with the experimental data. In figure 3(b) the resulting effective refractive indexes as a function of wavelength are plotted and they exhibit a normal dispersion. The obtained results show that $n_{\text {eff }}$ decreases when the porosity of the AAO template is increased with values ranging from $\sim 1.5$ to $\sim 1.42$. This behaviour is expected since a larger porosity means more volume of the sample occupied by air. Therefore, its effective refractive index should be lower and closer to the air value.

\subsection{AAO intrinsic refractive index}

The calculation of the intrinsic AAO refractive index was performed by applying the Maxwell-Garnett homogenization theory $[1,30]$. It is better suited than Bruggeman's model to this kind of system due to the well-separated optical domains defined by the AAO template (air filled nanopores + AAO) and the predominance of one of the constituents (AAO). The model assumes that as the structures (nanopores) present typical sizes far below the light wavelength at normal incidence, the permittivity of the whole system can be understood as an effective permittivity resulting from the combination of the constituents' ones, preponderating the contribution of the more extended medium defined as the host.

$$
\varepsilon_{\mathrm{eff}}=\varepsilon_{\mathrm{h}} \frac{1+\eta \Gamma}{1-\Gamma} \quad, \quad \Gamma=f_{\mathrm{g}} \frac{\varepsilon_{\mathrm{g}}-\varepsilon_{\mathrm{h}}}{\varepsilon_{\mathrm{g}}+\eta \varepsilon_{\mathrm{h}}},
$$

Equation (2) presents the Maxwell-Garnett model for only one guest material, which is air in our case. The effective, the host, and the guest electrical permittivities are represented by $\varepsilon_{\text {eff }}, \varepsilon_{\mathrm{h}}$, and $\varepsilon_{\mathrm{g}}$, respectively; $f_{\mathrm{g}}$ is the filling ratio of the guest medium and $\eta$ is the screening factor. This last term is related to the scattering induced by the shapes of the inclusions. By a straightforward operation, a quadratic equation for the host
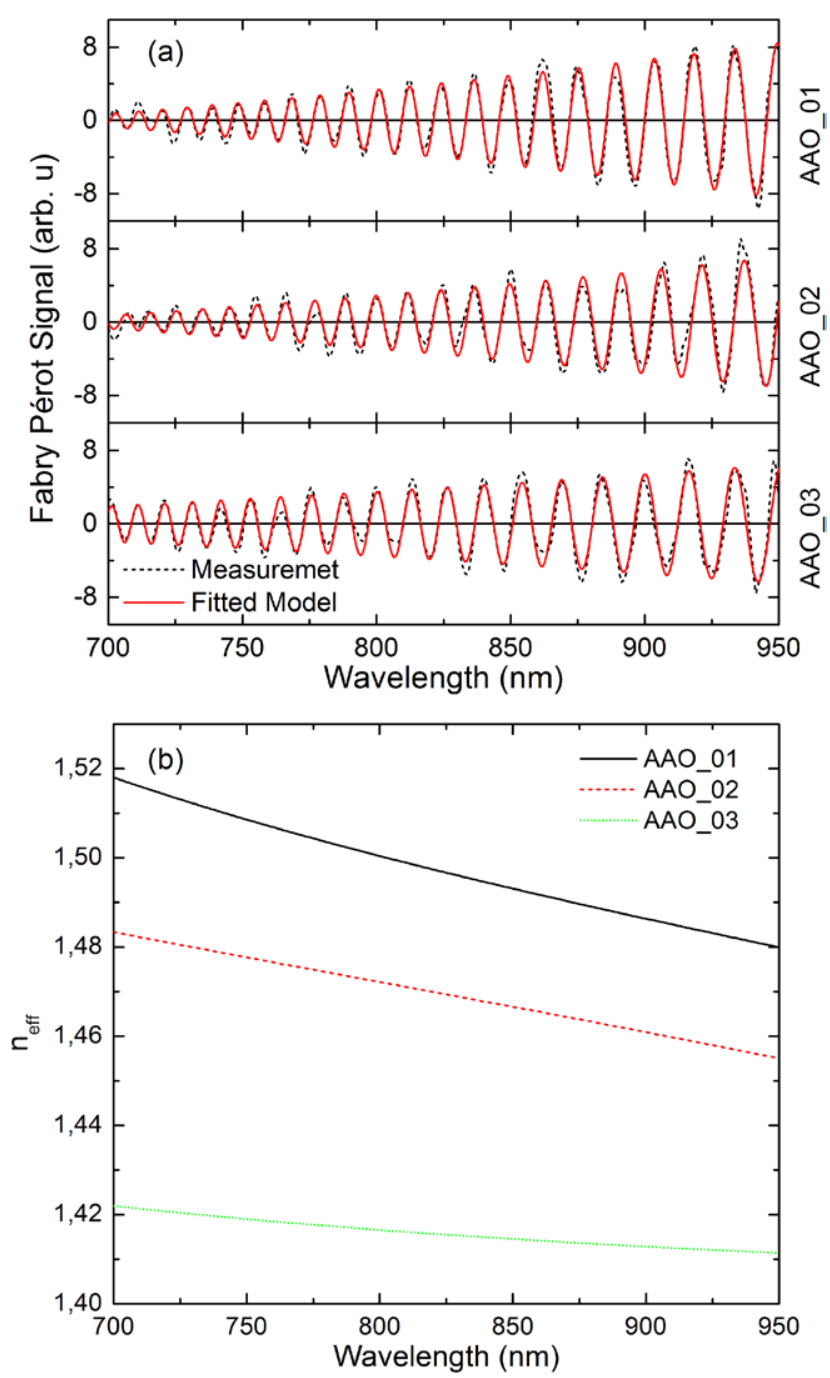

Figure 3. (a) Fitting model (continuous red line) compared with the experimental data (dashed black line) for the three fabricated templates, AAO_01, AAO_02, and AAO_03. (b) Effective refractive indexes constructed from the fitted Sellmeier's parameters for the three templates, AAO_01 (black line), AAO_02 (red line), and AAO_03 (green line).

medium electrical permittivity can be written, as illustrated in equation (3).

$\varepsilon_{\mathrm{h}}^{2}\left(\eta-\eta f_{\mathrm{g}}\right)+\varepsilon_{\mathrm{h}}\left[\varepsilon_{\mathrm{g}}+\eta f_{\mathrm{g}}-\varepsilon_{\mathrm{eff}}\left(f_{\mathrm{g}}+\eta\right)\right]+\varepsilon_{\mathrm{eff}} \varepsilon_{\mathrm{g}}\left(f_{\mathrm{g}}-1\right)=0$.

At normal incidence, where the nanopores ('air nanowires') are parallel to the light wave vector, the $\eta$ value is 1 . Also, $\varepsilon_{\mathrm{g}}$ is 1 and $\varepsilon_{\text {eff }}$ takes the values previously calculated through the Sellmeier fitting of the Fabry-Pérot response $\left(\varepsilon_{\mathrm{eff}}=n_{\mathrm{eff}}^{2}\right)$, leaving only the filling ratio $\left(f_{\mathrm{g}}\right)$ as incognita to determine the intrinsic permittivity of the AAO. This parameter was obtained after a careful and detailed analysis of the SEM images taken for each template. In figure 4(a) the final binary image filtered from the AAO_03 SEM micrograph is presented, showing the AAO regions in black and the air-filled nanopores in white. After this first step, the filling ratios' frequency distributions were extracted by measuring the $d_{\mathrm{p}}$ of each nanopore in the binary image and calculating the average distance $D$ between 

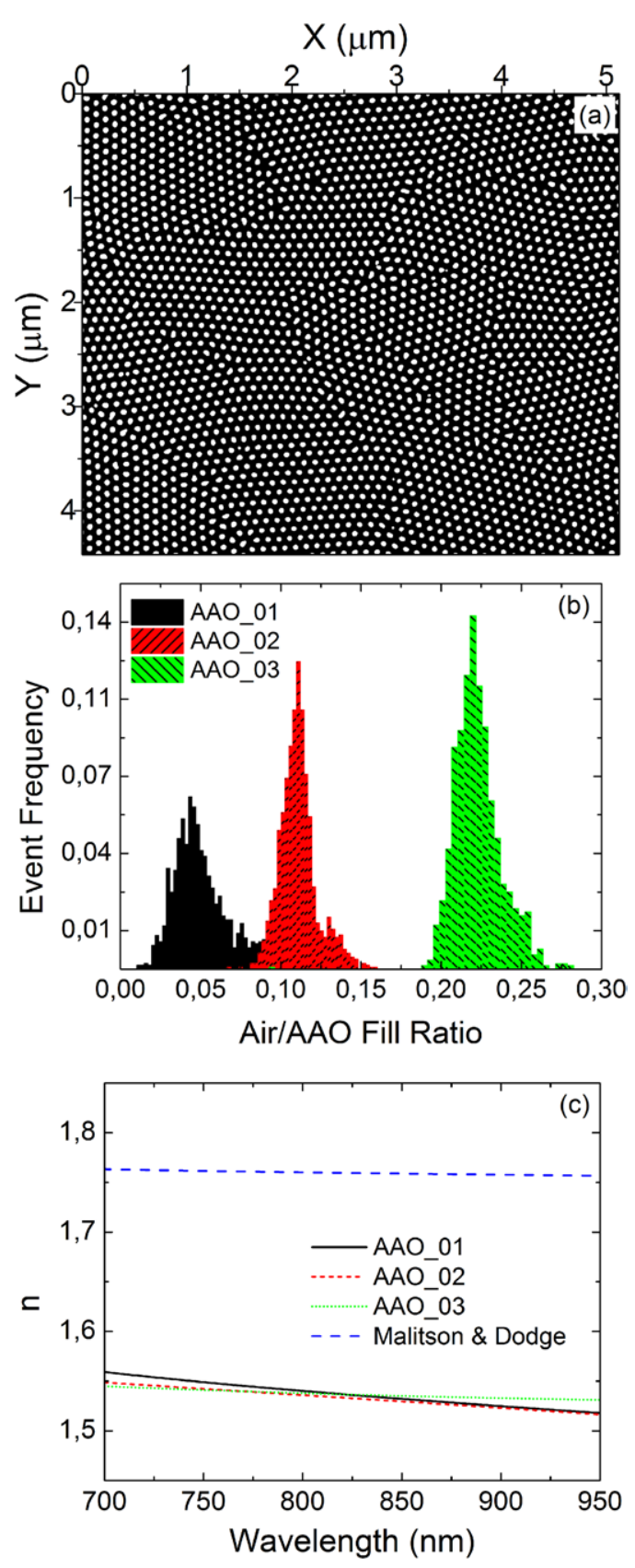

Figure 4. (a) Selected binary image of the nanopores' structures obtained from the SEM micrograph of sample AAO_03.

(b) Frequency distributions of the templates' filling ratios for samples AAO_01 (black), AAO_02 (red), and AAO_03 (green). (c) Calculated intrinsic refractive indexes for the $\mathrm{AAO}$ obtained from templates AAO_01 (black line), AAO_02 (red line), and $\mathrm{AAO}$ 03 (green line), and the handbook $\mathrm{Al}_{2} \mathrm{O}_{3}$ refractive index value (blue dashed)

the first nanopore neighbours. This leads to the filling ratio of each cell by assuming a hexagonal lattice order following the expression $f_{\mathrm{g}}=\left(4 \pi^{2} / 3\right)^{1 / 2} \cdot\left(d_{\mathrm{p}} / 2 D\right)^{2}$.

The resulting filling ratios' frequency distributions are presented in figure 4(b) for the three fabricated templates. It is clearly shown that the pore widening process leads to an increase in the nanopores' filling ratios. Their average values obtained for samples AAO_01, AAO_02 and AAO_03 are $\sim 0.05, \sim 0.11$, and $\sim 0.22$, respectively. Finally, the intrinsic
AAO refractive index was calculated for each wavelength by using equation (3) and the frequency distributions of the filling ratios. Thus, the obtained values take into account not only the average behaviour but also the whole information contained in the SEM micrographs. Figure 4(c) illustrates the intrinsic AAO refractive index as a function of wavelength for the three studied templates showing normal dispersion and almost coincident values in the whole analysed spectrum range. This fact is by itself a confirmation that the presented calculation method for the intrinsic refractive index is consistent and leads to the same properties of the underlying AAO material. In addition, the commonly used $\mathrm{Al}_{2} \mathrm{O}_{3}$ handbook refractive index value [23] is shown for comparison. We would like to highlight a reduction of $\sim 22 \%$ in the intrinsic AAO refractive index. This lower value can be understood due to the amorphous nature of the fabricated AAO [31]. Even when it is compared with the values measured for nanocrystalline/amorphous sputter-deposited alumina, our result is still below $(\sim 5 \%)$ the thin film's refractive index $(\sim 1.63)$ [24]. These differences are critical when designing and simulating the final response of an AAO template-based optical metamaterial, especially when dealing with plasmonic systems due to the extreme sensitivity of the plasmons to differences in the surrounding dielectric refractive index.

\section{Conclusions}

Different AAO nanoporous templates were fabricated by following the standard two-step anodization process and a chemical etching procedure to change their relative porosities. Their modification leads to a change in the AAO template's effective refractive index without changing the intrinsic AAO optical properties. The structural properties of the nanoporous templates (lattice parameter, nanopore diameter, template thickness) were studied by a careful and detailed analysis of the SEM images taken for each sample. By performing standard spectroscopic light transmission measurements, the effective refractive index for each sample was calculated by directly fitting the Fabry-Pérot signal of each transmittance spectrum. This fitting takes into account the dispersive properties given by the Sellmeier model. Finally, the intrinsic AAO refractive index was extracted by the Maxwell-Garnett homogenization theory, using the effective index and the structural properties of the templates. The obtained value is quite lower ( 22\%) than the commonly used $\mathrm{Al}_{2} \mathrm{O}_{3}$ refractive index handbook value and is also below $(\sim 5 \%)$ those measured in alumina thin films. These results are critical and must be taken into account when designing and simulating AAO-based optical metamaterials, especially when dealing with plasmonic systems.

\section{Acknowledgments}

The authors acknowledge the financial support from Spanish MINECO under project FIS2013-45469-C4-4-R and FCT of Portugal under projects UID/EEA/50014/2013 and UID/NAN/50024/2013, and NORTE-07-0124FEDER-000070 -Multifunctional Nanomaterials and 
NORTE-07-0124-FEDER-000058, which are financed by the North Portugal Regional Operational Programme (ON.2-O Novo Norte), under the National Strategic Reference Framework (NSRF), through the European Regional Development Fund (ERDF), and by national funds, through the Portuguese funding agency, FCT. A Hierro-Rodriguez and J M Teixeira acknowledge support from FCT of Portugal (Grants SFRH/ BPD/90471/2012 and SFRH/BPD/72329/2010, respectively) and POPH/FSE programs.

\section{References}

[1] Cai W and Shalaev V 2010 Optical Metamaterials, Fundamentals and Applications (New York: Springer)

[2] Sharma B, Frontiera R R, Henry A I, Ringe E and Van Duyne R P 2012 Mater. Today 15 16-25

[3] Soukoulis C M, Kafesaki M and Economou E N 2006 Adv. Mater. 18 1941-52

[4] Soukoulis C M, Linden S and Wegener M 2007 Science 315 47-9

[5] Poddubny A, Iorsh I, Belov P and Kivshar Y 2013 Nat. Photon. 7 948-57

[6] Zhang X and Liu Z 2008 Nat. Mater. 7 435-41

[7] Fang N, Lee H, Sun C and Zhang X 2005 Science 308 534-7

[8] Hao F, Sonnefraud Y, Van Dorpe P, Maier S A, Halas N J and Nordlander P 2008 Nano Lett. 8 3983-8

[9] Kabashin A V, Evans P, Pastkovsky S, Hendren W, Wurtz G A, Atkinson R, Pollard R, Podolskiy V A and Zayats A V 2009 Nat. Mater. 8 867-71

[10] Pendry J B, Schurig D and Smith D R 2006 Science 312 1780-2

[11] Cai W, Chettiar U K, Kildishev A V and Shalaev V M 2007 Nat. Photon. $1224-7$

[12] Sousa C T, Leitao D C, Proenca M P, Ventura J, Pereira A M and Araujo J P 2014 Appl. Phys. Rev. 1031102
[13] Sousa C T, Leitao D C, Proenca M P, Apolinario A, Correia J G, Ventura J and Araujo J P 2011 Nanotechnology 22315602

[14] Mátéfi-Tempfli S, Mátéfi-Tempfli M, Vlad A, Antohe V and Piraux L 2009 J. Mater. Sci.: Mater. Electron. 20 249-54

[15] Neumann R F, Bahiana M, Allende S, Altbir D, Görlitz D and Nielsch K 2015 Nanotechnology 26215701

[16] Abreu Araujo F, Piraux L, Antohe V A, Cros V and Gence L 2013 Appl. Phys. Lett. 102222402

[17] Hauet T et al 2014 Phys. Rev. B 89174421

[18] Piraux L, Renard K, Guillement R, Mátéfi-Tempfli S, Mátéfi-Tempfli M, Antohe V A, Fusil S, Bouzehouane K and Cros V 2007 Nano Lett. 7 2563-7

[19] Antohe V A et al 2009 Appl. Phys. Lett. 94073118

[20] Wesche M, Hüske M, Yakushenko A, Brüggemann D, Mayer D, Offenhaüsser A and Wolfrum B 2012 Nanotechnology 23495303

[21] Custodio L M, Sousa C T, Ventura J, Teixeira J M, Marques P V S and Araujo J P 2012 Phys. Rev. B 85165408

[22] Leite I T, Hierro-Rodriguez A, Silva A O, Teixeira J M, Sousa C T, Fernandez-Garcia M P, Araujo J P, Jorge P A S and Guerreiro A 2013 Phys. Status Solidi-R 7 664-7

[23] Weber M J 1986 Handbook of Laser Science and Technology vol 4 (Boca Raton, FL: CRC Press)

[24] Kischkat J et al 2012 Appl. Opt. 51 6789-98

[25] Masuda H and Fukuda K 2001 Science 268 1466-8

[26] Lutich A A, Gaponenko S V, Gaponenko N V, Molchan I S, Sokol V A and Parkhutik V 2004 Nano Lett. 4 1755-8

[27] Li Y B, Zheng M J, Ma L and Shen W Z 2006 Nanotechnology 175101

[28] Sellmeier W 1871 Ann. Phys. Chem. 219 272-82

[29] Tatian B 1984 Appl. Opt. 23 4477-85

[30] Maxwell-Garnett J C 1904 Phil. Trans. R. Soc. Lond. $203385-420$

[31] Leitao D C, Ventura J, Sousa C T, Pereira A M, Sousa J B, Vazquez M and Araujo J P 2011 Phys. Rev. B 84014410 